\title{
Enhanced SDL Subset for the Design and Implementation of Java-Enabled Embedded Signalling Systems ${ }^{\star}$
}

\author{
Christoforos Kavadias ${ }^{1 \star \star}$, Bernard Perrin ${ }^{2}$, \\ Vangelis Kollias ${ }^{1}$, and Michael Loupis ${ }^{3}$ \\ 1 TELETEL SA; 124, Kifissias Avenue, Athens, Greece \\ C.Kavadias@teletel.gr V.Kollias@teletel.gr \\ 2 CSEM: Centre Suisse d'Electronique et de Microtechnique \\ Rue Jaquet-Droz 1, CH-2007 Neuchâtel \\ bernard.perrin@csem.ch \\ 3 SOLINET GmbH Solutions for Innovative Networks \\ Mittlerer Pfad 26, 70499 Stuttgart, Germany M.Loupis@SOLINET.com
}

\begin{abstract}
This paper proposes an enhanced SDL subset for the implementation of embedded signalling systems and describes a novel methodology for its translation to Java based applications. Although SDL is widely used in the telecommunications field, it is also now being applied to a diverse number of other areas ranging over aircraft, train control, medical and adaptive systems. Embedded signalling systems form a class of applications in the telecommunications field with specific requirements within the SDL domain. Although SDL is commonly used to specify embedded signalling systems, typically it is only a subset of the SDL language that is relevant. Within this contribution the key requirements of embedded signalling systems are identified and an enhanced subset of SDL 2000 is proposed for their specification and implementation. Additionally, this paper proposes a methodology for the translation of SDL systems based on this enhanced SDL subset, to Java applications using an SDL to Java Translator for supporting emerging Java based applications. The current proposal does not contradict with any SDL specification, but instead it is complementary to current SDL standards considering together design and implementation requirements of embedded signalling systems.
\end{abstract}

\section{Introduction}

Nowadays, we are experiencing a technology revolution in the telecommunications field. Modern telecommunication systems (including embedded signalling systems) are characterized by dynamic expansion and heterogeneous nature due to the interconnection of components having diverse technical characteristics.

\footnotetext{
* This work is partially supported by the EC under the IST-65002 REMUNE project.

${ }^{\star \star}$ Correspondence author.
} 
With this level of continuous technical evolution it is essential that simple, efficient and highly reliable telecommunications systems are required before network operators offer new services to the users.

Currently in the market, there are various tools available for the specification of event based embedded signalling systems based on ITU-T SDL[1, 2, 3]. Although SDL is widely used in the telecommunications field, it is also now being applied to a diverse number of other areas ranging over aircraft, train control, medical and packaging systems. The expansion of SDL beyond the telecommunications field has led to the development of complex SDL based tools, supporting numerous of SDL features and keywords. Thus, the more complex the design of a signalling system is, the more complex is its implementation. Although SDL is commonly used to specify embedded signalling systems, typically it is only a subset of the SDL language that is relevant. From an implementation point of view and concerning platforms that support the execution of SDL based systems, simplicity is required in order to provide higher reliability and efficiency.

On the other hand, Java technology has become a significant force stimulating the evolution of embedded systems. The simplicity and platform-independence of the language attracted developers who struggled with portability issues and project deadlines. Since that time, developers in the embedded device market have come to understand and appreciate the benefits of the Java platform.

Sect. 2 of this paper defines a meaningful SDL subset for the support of embedded signalling systems design and implementation and Sect. 3 proposes the necessary enhancements to this SDL subset in order to satisfy the requirements of embedded signalling systems design and implementation. The aim of the enhanced SDL subset proposed in this paper is to provide simplicity and efficiency at the design level, something that leads to the implementation of robust and highly reliable telecommunication systems. Because Java is widely used in the implementation of embedded telecommunications system, Sect. 4 provides a methodology for the translation of SDL systems based on this enhanced SDL subset, to Java applications using an SDL to Java Translator for supporting emerging Java based applications.

\section{Definition of a Meaningful SDL Subset for the Support of Embedded Signalling Systems Design and Implementation}

Embedded signalling systems have the general characteristics mentioned in the introduction. User demands in terms of improved service quality, high-reliability, efficiency, increased bandwidth and advanced telecommunication services are gradually growing. As new technologies are integrated, customers expect and demand that personal communications will be capable of managing and delivering a much wider range of information services to the global market.

Given the speed and efficiency of evolution of new technologies and the continually growth of user requirements, in terms of improved service quality, highreliability, efficiency and unlimited bandwidth, the rapid increase of the existing 
telecommunication networks' size and complexity is unavoidable. New requirements abound, driven by a large and diverse user population and the progress of technology. Furthermore, the convergence of various networks for the provision of advanced network services and applications is growing rapidly.

With this level of continuous technical evolution it is essential that simple, efficient and highly reliable telecommunications systems are required before network operators offer new services to the users.

Embedded signalling telecommunication systems have specific characteristics and address areas with unique requirements. They are based on Finite State Machines (FSMs), which means that they have defined states from where they can receive and send signals. Additionally, they consist of processes that send signals to each other based on a peer architecture. When waiting for an input signal a process is idle, which is a key difference to other (batch) applications. Such systems have specific requirements within the SDL domain and commonly only a subset of SDL is relevant.

In the work presented in this paper we have identified the requirements of embedded signalling systems ranging from embedded telecommunication protocols (SS7, V5, ISDN etc.) to other industrial systems. This work is a part of the work that is being performed within the context of the REMUNE IST project $[5,6]$. The key features required from the current version of SDL to support the design and implementation of embedded signalling systems are:

- Data types, constants, variables

- Signals

- Connections between FSMs

- Decisions

- Procedures

- FSMs

- Timers

- Assignments

New features that have been added in the latest versions of SDL (including object-oriented features) are not of great importance for signalling systems, because no added value capabilities are provided for the design and implementation of such systems.

\section{Enhancements to the SDL Subset}

The current version of SDL provides the features that support design and implementation of embedded signalling systems. However, there is a set of requirements that are not directly satisfied by the current SDL features. ${ }^{1}$ These requirements are classified in three main areas:

\footnotetext{
${ }^{1}$ Editorial note: In the editor's opinion the issue is mainly the current lack of tool support: an issue which the REMUNE project is addressing.
} 
- Efficient management of resources: such as CPUs, memory especially in embedded systems, simple memory operations for dynamic memory allocation;

- Design in SDL to map the implementation constraints: such as the ability to assign to a variable a specific value centrally (at a system level) without having to assign the desired values "hard-coded";

- Need for more modularity: such as the ability to define substates within states in order to provide more simplicity and maintainability.

The following paragraphs analyze this set of requirements and propose a set of additional SDL keywords to support these concepts within the SDL domain. The current proposal does not contradict with any SDL specification, but instead it is complementary to current SDL standards considering together design and implementation requirements of embedded signalling systems.

\subsection{Enhancements of SDL for Efficient Resource Management}

As far as the efficient resource management is concerned, telecommunication systems and especially embedded systems are characterized by limited resources including CPU and memory. Although SDL can be used as a specification and description language for embedded signalling systems, there are tools that support the execution of SDL based systems directly to the target. The efficient resource management is not a main requirement for the description and design of systems using SDL, but in the case of SDL based implementations this is no longer true.

Currently, SDL supports arrays for the declaration of variables that belong to a specific group of variables of the same type. However, arrays are not applicable in areas such as embedded systems, where the resources are limited and efficient memory management is required. Therefore, simple memory operations for dynamic memory allocation are needed.

This contribution proposes some additional SDL keywords that can be used for the efficient memory management in embedded signalling systems. The proposed concepts are coming directly from other programming languages such as ANSI C, which supports a set of memory operations for efficient memory handling. The proposed SDL keywords and a short description for each one is provided below:

- PUSH - Handling Lists' variables. The PUSH function is used in order to add elements to a list.

- POP - Handling Lists' variables. The PUSH function is used in order to read and remove elements from a list.

- PUSH_FRONT - Handling Lists' variables. Same operation as PUSH.

- PUSH_BACK - Handling Lists' variables. The PUSH_BACK function is used in order to add elements to the end of a list.

- POP_FRONT - Handling Lists' variables. Same operation as POP

- POP_BACK - Handling Lists' variables. The POP_BACK function is used in order to read and remove elements from the end of a list. 
- ELEMENT_FRONT - Handling Lists' variables. The ELEMENT_FRONT function is used in order to read elements (without removing them) at a given index in a list (start counting from the beginning of a list).

- ELEMENT_BACK - Handling Lists' variables. The ELEMENT_BACK function is used in order to read elements (without removing them) at a given index in a list (start counting from the end of a list).

- REMOVE_FRONT - Handling Lists' variables. The REMOVE_FRONT function is used in order to delete elements from the beginning of a list.

- REMOVE_BACK - Handling Lists' variables. The REMOVE_BACK function is used in order to delete elements from the end of a list.

\subsection{Enhancements of SDL to Map Implementation Constraints}

As far as the design in SDL to map the implementation constraints is concerned, embedded signalling systems and especially switching systems require a mechanism for the management of variables that the system behavior depends on. A specific scenario, for example, may be for a protocol layer to be dynamically changed from user to network mode. Such a mechanism will allow the changes of specific variables within SDL based systems to be performed centrally, even during runtime (for executable SDL based systems).

The concept for addressing this requirement is based on the Management Information Bases (MIBs), which are a collection of definitions, which define the properties of the managed object within the device to be managed. Every managed device keeps a database of values for each of the definitions written in the MIB. It is not the actual database itself - it is implementation dependent.

The current version of SDL does not support a keyword that allows the efficient management and configuration of significant SDL variables in SDL based implementations that may affect the behavior of an SDL based system. The proposed SDL keyword as well as a short description and an example is provided below:

- MIB - Variables' update declaration. This special variables' declaration is used in order to allow the user to have the ability of assigning a different value to a variable, even during the systems' execution. From the standard value assignment, the MIB assignment differs only at the end of the assignment where the keyword MIB is added.

\subsection{Enhancements of SDL for more Modularity}

As far as the need for more modularity is concerned, most of embedded signalling systems in the telecommunications world are of high complexity and therefore the difficulty in the maintenance of their implementations increases, having as a result the increase of costs. Although SDL is a modular language, the telecommunication industry asks for special features that will allow advanced modularity of SDL based systems. Within this contribution, the concept of nested states is proposed. The proposed SDL keywords that will allow this are as follows: 
- SUBSTATE - Begin of nested state

- ENDSUBSTATE - End of nested state

These keywords are used for nesting states into other states. This concept is mostly covered by SDL 2000, which provides the concept of composite states. However, the composite states concept is more complex than the one proposed in this paper, since it incorporates additional functionality. The current proposal does not contradict with the composite states concept, but instead it is used to serve as the part of the composite state functionality that has to do only with nesting of states in other states.

The reason for introducing these keywords is again that of simplicity.

The paragraphs above provide a description of the enhancements that should be performed to the current SDL keywords for satisfying the requirements that derived directly from the telecommunications world for the design and implementation of embedded signalling systems. The following section provides a methodology for the translation of the enhanced SDL subset to Java using an SDL to Java Translator for supporting emerging Java based applications.

\section{Mapping SDL Designs to Implementation Platforms (SDL to Java Translator)}

Java technology has become a significant force stimulating the evolution of embedded systems. The simplicity and platform-independence of the language attracted developers who struggled with portability issues and project deadlines. Since that time, developers in the embedded device market have come to understand and appreciate the benefits of the Java platform.

The aim of the enhanced SDL subset proposed in this paper is to provide simplicity and efficiency at the design level, something that leads to the implementation of robust and highly reliable telecommunication systems. Because Java is widely used in the implementation of embedded telecommunications system, this section proposes a methodology for the translation of SDL systems based on this enhanced SDL subset, to Java applications using an SDL to Java Translator.

\subsection{Working Assumptions}

Today, there are more and more small-embedded systems supporting a Java Virtual Machine (JVM). The Sdl2Java translator has been designed for such systems. So we considered as an important constraint to keep the Java generated code as simple as possible. Although tempted, we avoided using complex enhancements such as Java Beans or Enterprise Java Beans (EJB).

A second advantage coming with this simplicity is the production of fast code, having a short response time and prepared for real-time (RT) applications. Java is usually considered slow. However, this is only true when we use the above enhancements or (for a GUI) the Abstract Windowing Toolkit (AWT). 
Another important aspect we considered prior to our developments is the portability of the translator itself. Portability confers an evident added value to one tool when you can use it on diverse platforms.

\subsection{Tools Choice}

The choice was made between two families of tools:

- lex/yacc (flex/bison)

- JavaCC[7]/JJTree-JTB[8]

The first one produces C-code, which can be considered as portable. The second one generates Java code, which by the very essence of Java is portable at an executable level. We found the following advantages to the second family and therefore selected it:

1. JJTree or JTB (Java Tree Builder) are automatically adding the parse tree building actions.

2. The generated parser is in the same language as the code to be produced by the translator. It is certainly an advantage to think in a single language (Java) rather than two (C+Java).

\subsection{The SDL Package}

In order to keep the generated code as short as possible, we based it on an existing and hand-written package, the SDL package. All the base classes instantiated within the generated code are defined in this package. Some of them are represented in Fig. 1. The generated code always instantiates two classes: an SdlSystem class and an Engine class. The last step in the generated code of the main() method is to invoke the exec() method of the Engine class with a reference to the SdlSystem.

As shown below, there are two kinds of engines, both derived from the Engine class. A basic method of this ancestor class is OneTickExecute() and differently called by the child engines. The SimulatorEngine children invokes this method each time the user would like to execute a step or repeatedly when after a run command is issued. The RealTimeEngine invokes the OneTickExecute() method at a given timer period. If the previous call is not returned before the timer expires, the system will stop and display an error (tick time interval is too short).

The SimulatorEngine class includes a very primitive user interface supporting commands such as:

- go [ticks]: run the simulator for a given number of ticks (no given value means forever)

- step: run the simulator for 1 tick (equivalent to "go 1") 


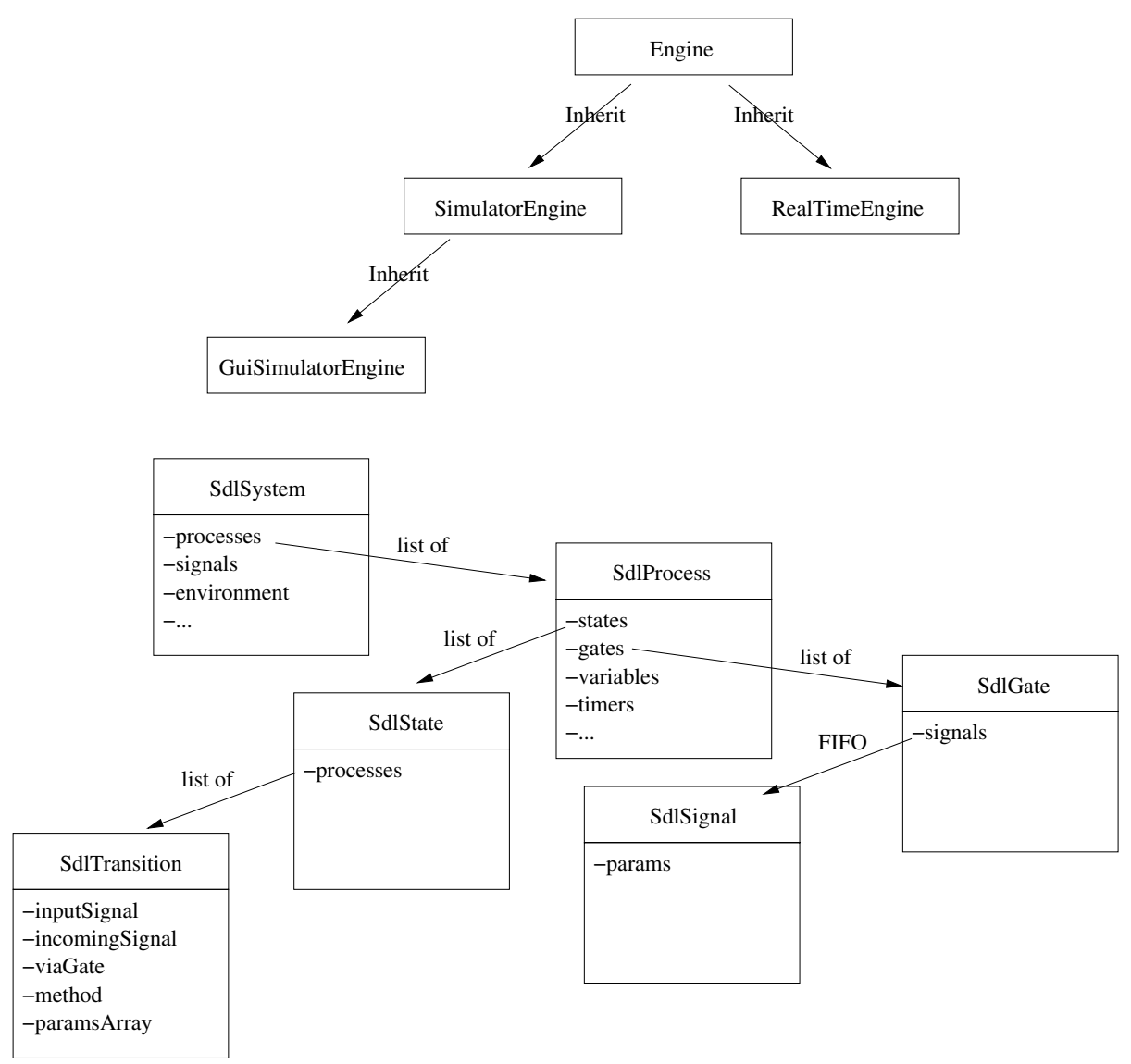

Fig. 1. Simplified view of some of the Sdl package classes

- batch file: execute one file in batch mode. The file must end with < exit> to return to the normal mode

- output $<$ sig $><$ proc $><$ gate $>$ : send a signal $<$ sig $>$ from the environment to process $<$ proc $>$ via gate $<$ gate $>$

- var: display all variables of all processes

- gate: display gates in each process and their contents

- state: display the current state of each process

- trace [value]: display or set the trace level (higher -> more debug messages)

- time [value]: display or set the current time in number of ticks

- exit | quit: quit the simulator

The GuiSimulatorEngine redirects the IO stream to a socket server, where the GUI is located. A web based GUI has been built and is shown below in Fig. 2. 


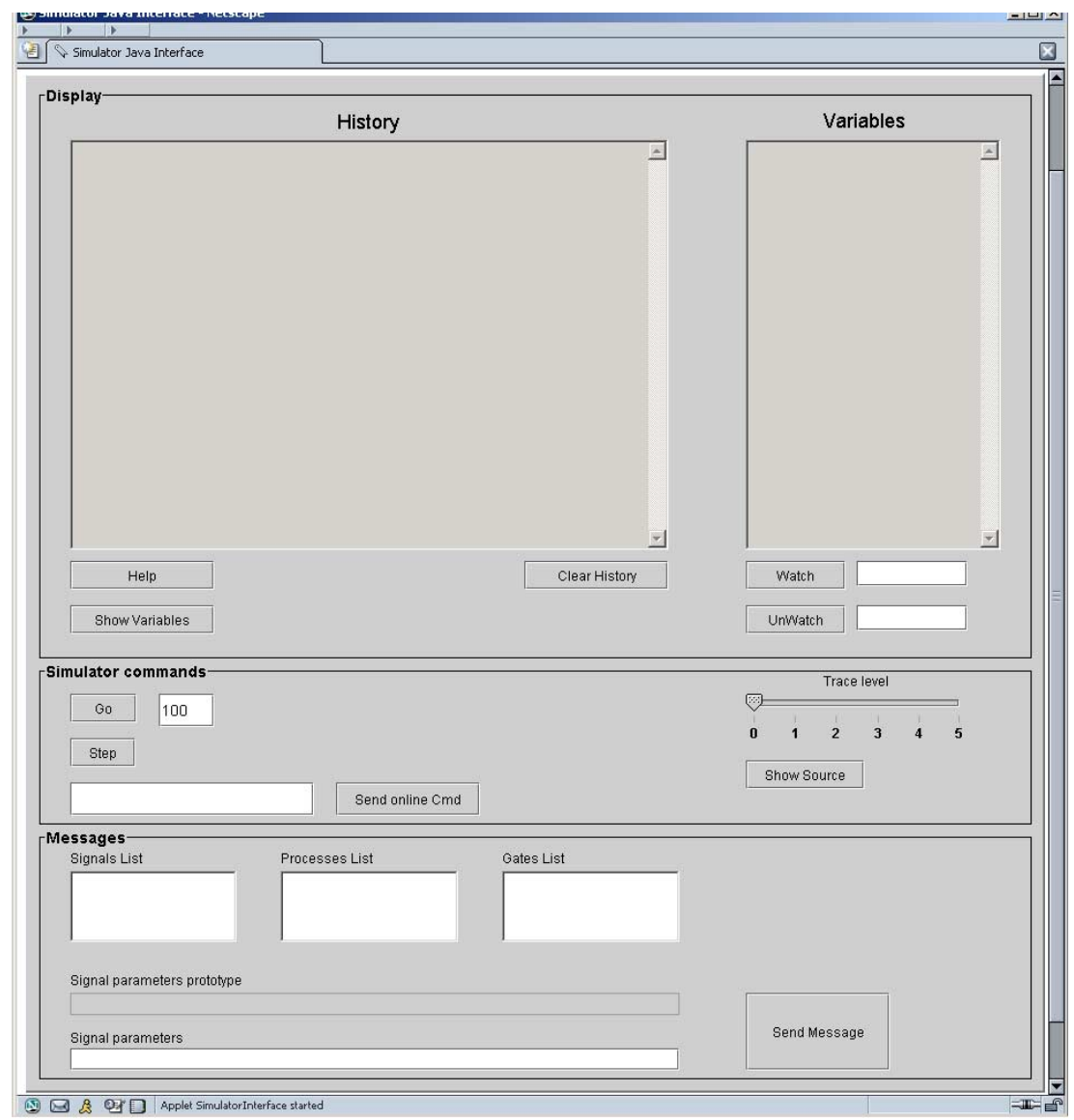

Fig. 2. Web based GUI

As shown in Fig. 1, an SDL system is constituted from a list of SdlProcess (java LinkedList). Each SdlProcess holds among others a list of SdlState, where each one is defined by a list of SdlTransition. Each transition is characterized by the conditions able to start it and the associated methods to run, with their parameters.

The timers are not shown but are simple entities, decremented, when running, after each tick. A timer occurrence is managed in the same way as a sent signal.

OneTickExecute() calls the SubTickExecute() method as long as transitions are occurring. This last method is the kernel of the Engine: its basic task is to look through all gates in all processes and extract the queued signals, if any, to start the necessary transitions. 


\subsection{The Generated Code}

Before generating the code, the SDL description is parsed. A parse tree is automatically built, thanks to JTB. A template visitor method is also generated by JTB for each node and this allows a recursive visit of the entire tree. After the parse tree has been built, two visits to the tree are necessary, each one with a different goal. The first pass is dedicated to discover the system name, the signals, the user defined types (syntype), the states, etc. To summarize, everything that could be used and potentially be defined later on in the SDL pr file has to be detected during the first pass. Then during the second pass, it is already known even if appearing later in the source code.

During the two passes, an internal representation of the code to generate is built. This is based on the CodeGen package, which is sometimes close to the Sdl package presented in the previous section.

Then the design has to be flattened. When an SDL process is defined within a block, which in turn could be in a block, the final representation will just be one process. In the Java generated code the block instance hierarchy is used for the name preamble of the process. This operation makes the signal routing much simpler. Only processes at the same level (the top level) appear in the system.

The generated code is rather simple to read and understand. It consists of Sdl package component instantiations and by the relations between them. One Java procedure is generated per each process. The Engine at each transition of the concerned process invokes it. The process initialization is the first transition of any process. The procedure returns a pointer to the next state. Our first idea was to generate one procedure per transition. However, this is not possible since SDL has a JOIN statement allowing jumping from one transition body to any other one. In other words, this SDL statement can lead to a non structured program. On the other hand, a Java program is, by its language definition, always structured. Introducing a switch statement in an infinite loop with break statements has solved this problem.

We also considered the solution of starting one thread per process, and to realize the signal communication through channels via message queues. However, we were soon convinced that this solution would not require less overhead and will be more difficult to control in order to be compliant with the SDL specification (same signal scheduling leading to the same potential deadlocks).

\subsection{The Enhanced SDL Subset for Sdl2Java}

We enhanced the SDL subset with the following keywords:

- \#JAVA_CODE, \#JAVA_ENDCODE

- \#JAVA_HEADING, \#JAVA_ENDHEADING

- \#SDL()

The first two statements allow the possibility to introduce Java code directly in the SDL specification. This is particularly useful to call an already developed 
and tested Java method. The next two statements are mainly used to be able to include the package reference where the above used methods reside. The last keyword is used to pass an SDL variable to this Java code.

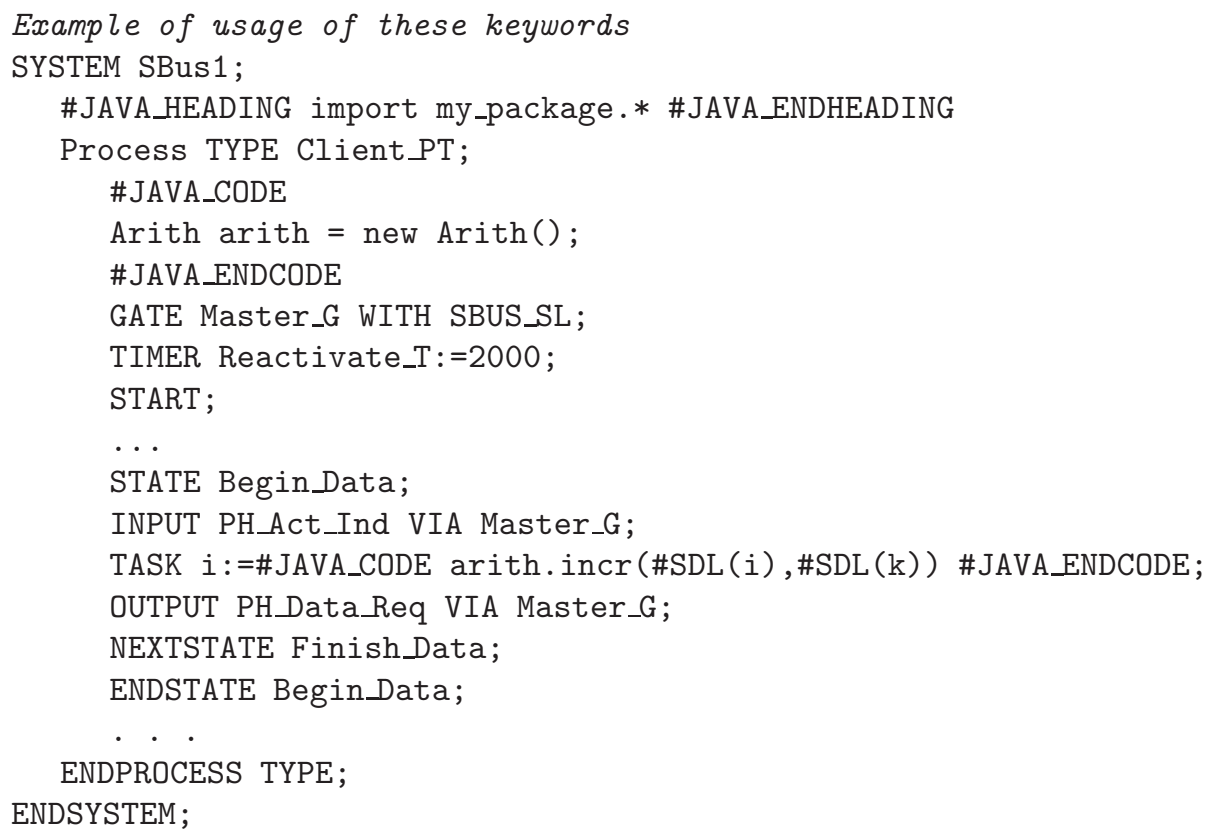

\subsection{The Trials and the TINI ${ }^{T M}$}

We used for our first trials the same machine to run the Sdl2Java translator and the generated code.

We first tried it on the following platforms:

- Sun/Solaris

- Mac/OsX

- PC/Windows

- PC/Linux

We had the same expected and correct results for all the 4 platforms. The test vehicle was a door access control. This small system is composed of two main instances: a door access controller, presenting the interface to the user, and a central controller, storing and checking to/from a database the access right of the different users presenting at the door.

We then decided to try the generated code on a small-embedded platform, the TINI $^{T M}$ board (see Fig. 3) from Dallas Semiconductors. Such a board is shown below. The Java generated \& compiled code and the Sdl package classes have to 


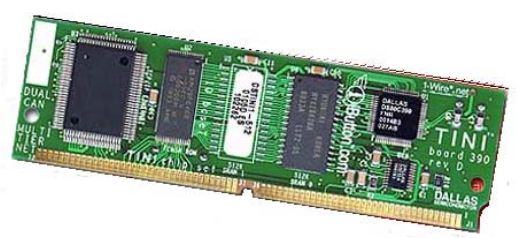

Fig. 3. TINI ${ }^{T M}$ board at SIMM card format

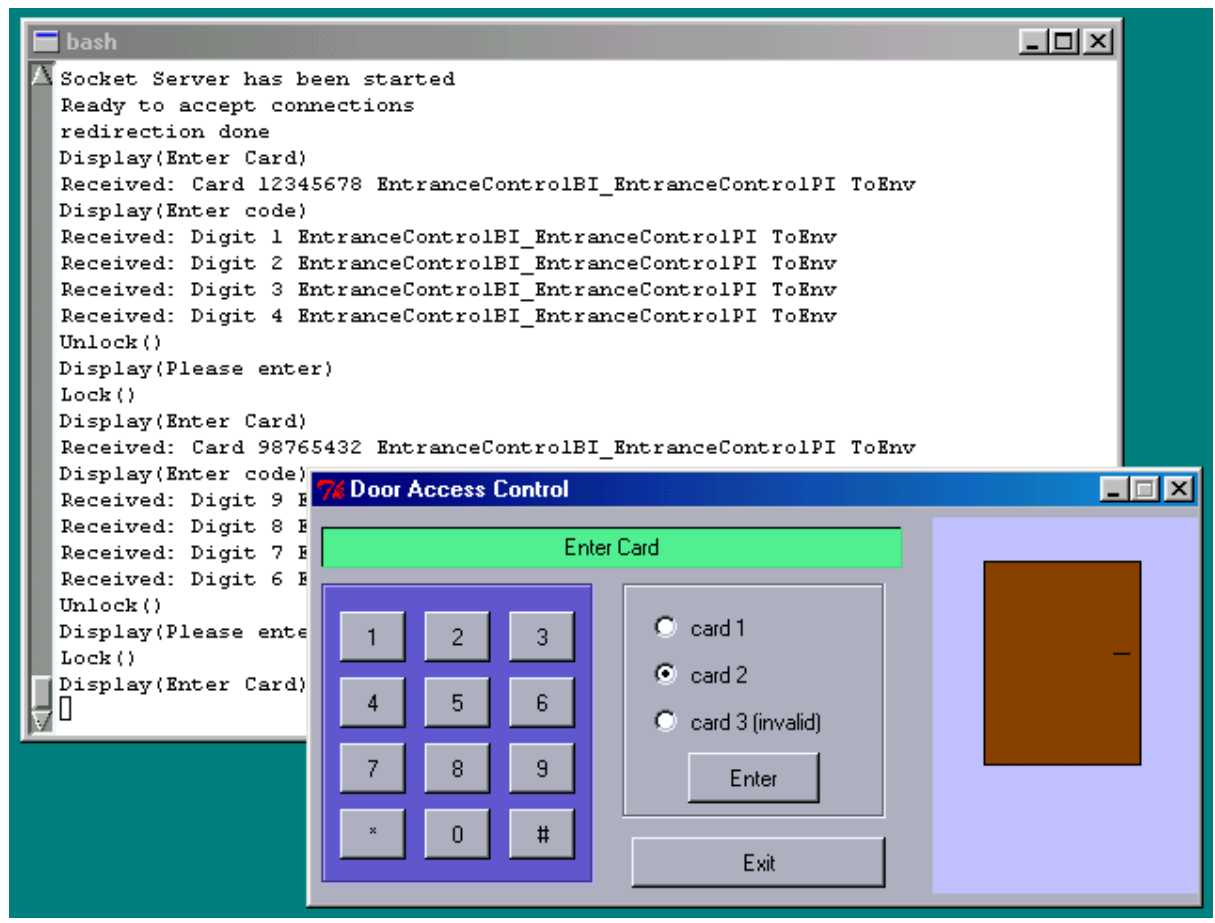

Fig. 4. Door Access Control GUI and log window on the TINI ${ }^{T M}$ board

be translated through a Dallas utility (TINIConvertor) before an execution on the TINI ${ }^{T M}$ JVM.

Until now, the classes' conversion works without any error and the tested system runs correctly. We had to rewrite the LinkedList class, which is not supported and find a workaround to a known bug of the TINI ${ }^{T M}$ : an invoke with a NULL argument produces a "null pointer exception" even if it works correctly on the other platforms.

Figure 4 shows a log window on the TINI ${ }^{T M}$ where the Door Access Control System has been run. A Tcl/Tk GUI has been built on a PC to converse with the above system environment. The closed door on the right symbolizes the door to open with the right code associated with a known card (card 1 or 2). 


\section{Conclusions}

The enhanced SDL 2000 subset proposed in this paper is relevant for the implementation of embedded signalling systems. With the selected subset and extensions proposed in this contribution, most signalling or embedded systems can be modeled, thus there is no need for further complexity to be introduced in the overall SDL specification, which is targeting additional domains as well. Additionally, the proposed SDL subset and the extensions provide simplicity and efficiency at the design level, something that leads to the implementation of robust and highly reliable telecommunication systems. The current proposal does not contradict any SDL specification, but instead it is complementary to current SDL standards considering together design and implementation requirements of embedded signalling systems.

Because Java is widely used in the implementation of embedded telecommunications system, this paper proposes a methodology for the translation of SDL systems based on this enhanced SDL subset, to Java applications using an SDL to Java Translator. The choices and assumptions made for the Sdl2Java development has now revealed their correctness. This tool is still under development and is not yet compatible with the proposed enhanced SDL subset. However, there is no intrinsic limitation in the existing software structure which would inhibit its introduction.

\section{References}

[1] ITU-T Recommendation Z.100: Specification and Description Language 138

[2] ITU-T Recommendation Z.105: SDL combined with ASN.1 modules 138

[3] ITU-T Recommendation Z.107: SDL with embedded ASN.1 138

[4] Study Group 17 web site: http://www.itu.int/ITU-T/studygroups/com17/languages

[5] IST-65002 REMUNE project deliverable D21 "Industrial Requirements Analysis" 139

[6] IST-65002 REMUNE project deliverable D22 "REMUNE Functional Specifications and Validation Scenarios" 139

[7] JavaCC web site: http://www.webgain.com/products/java_cc/ 143

[8] JTB web site: http://www.cs.purdue.edu/jtb/ 143 